\title{
Spin Polarons in Antiferromagnetic Planes
}

\author{
J. MORKOWsKI
}

Institute of Molecular Physics, Polish Academy of Sciences

M. Smoluchowskiego 17, 60-179 Poznań, Poland

Dedicated to Professor Józef Spatek on the occasion of his 60th birthday

In the lecture the theory of spin polarons is reviewed on the basis of its analogy with the theory of lattice polarons. The energy dispersion curve for a single polaron in a two-dimensional antiferromagnetic square lattice is calculated in the self-consistent Born approximation. Also in self-consistent Born approximation the energy of a pair of interacting spin polarons is calculated. The results of calculations for realistic values of parameters of the model lead to the conclusion that pairing of spin polarons is not a likely mechanism of superconductivity in cuprates.

PACS numbers: 71.27.+a, 72.10.Di, 74.20.Mn

\section{Introduction}

The problem of spin polarons had arisen as one of many ideas which, hopefully, could lead to an understanding of the phenomena of high temperature superconductivity. The mathematical foundation of the spin polarons concept is the so-called $t-J$ model [1], proposed years ago in a quite different context. In line with the general topics of the school - superconductivity in the past and at present - the actively pursued in the past decade problem of spin polarons will be recalled.

Spin polarons were studied starting from a simple heuristic model approach [2], refined over years (see e.g. [3]) and also by extended computer simulations [4].

There is no place here to review the quite extensive literature on the subject. In the present lecture we intend to concentrate on a particular approach which exposes approximations underlying the widely accepted results of the theory. The treatment here makes use of the well-elaborated procedures used years ago in the theory of phonon polarons. 
The stoichiometric parent compounds of the superconducting cuprates are antiferromagnets. It seems well established that a key role to their properties are played by the $\mathrm{CuO}_{2}$ planes, or sequences of pairs in the same compounds, which are relatively weakly coupled to their environment. The simplest model to simulate the $\mathrm{CuO}_{2}$ planes is to consider them as a system of virtually localized electrons on $\mathrm{Cu}^{2+}$ ions with their spins interacting like in the Heisenberg spin 1/2 antiferromagnets by an exchange interaction $J \boldsymbol{S}_{j} \boldsymbol{S}_{j}$. In doped or oxygen deficient non-stoichiometric superconducting compounds the $\mathrm{CuO}_{2}$ planes can be modelled as antiferromagnetic (AF) systems of spins $1 / 2$ (virtually localized electrons) and a number of holes-lattice sites void of a spin (electron). A hole can hop to a neighbouring site at an energy cost, equal roughly to $2 J$ in the first approximation, if only the Ising components of spins are considered. The energy cost of hopping a hole to a site distant by, say, $n$ lattice constants would be of the order of $2 n J$ due to a string of spins in "wrong" positions. In the Ising approximation the hole would be localized due to the high energy costs of hopping. However, due to the transverse components of spins in the exchange interactions there exist fluctuations of spins which highly reduce the prohibitive energy costs of a movement of a hole. These spin fluctuations in otherwise AF planes are described in terms of AF magnons. A motion of a hole is thus accompanied by a cloud of magnons, similarly as a motion of an electron in deformable lattice is accompanied by a cloud of phonons. Thus there is analogy to the well-known (lattice) polaron - a bound state of an electron with a cloud of phonons. The notion of spin polaron is introduced, meaning a bound state of charge carrier dressed in a cloud of virtual magnons.

The above intuitive picture will guide us in describing a formal theory. The starting point is the so-called $t-J$ model defined by the Hamiltonian [1]:

$$
\begin{aligned}
\mathcal{H}_{t-J} & =-\sum_{\langle i, j\rangle, \sigma} t\left(1-n_{i,-\sigma}\right) c_{i \sigma}^{+} c_{j \sigma}\left(1-n_{j,-\sigma}\right) \\
& +\frac{1}{2} J \sum_{\langle i, j\rangle}\left(s_{i} \cdot s_{j}-\frac{1}{4} n_{i} n_{j}\right)
\end{aligned}
$$

with the usual definitions $n_{j \sigma}=c_{j \sigma}^{+} c_{j \sigma}, s_{j}^{x}+\mathrm{i} s_{j}^{y} \equiv s_{j}^{+}=c_{j \uparrow}^{+} c_{j \downarrow}, s_{j}^{z}=$ $(1 / 2)\left(n_{j \uparrow}-n_{j \downarrow}\right), n_{j}=\sum_{\sigma} n_{j \sigma}$. The Hamiltonian (1) was derived [1] in the strong coupling limit $U \gg|t|$ as an approximate equivalent of the single band Hubbard Hamiltonian $\mathcal{H}=-\sum_{i, j, \sigma} t c_{i \sigma}^{+} c_{j \sigma}+U \sum_{i} n_{i \uparrow} n_{i \downarrow}$; some three-particle type terms were neglected. The first term in (1) describes correlated hoppings of electrons such that double occupancies of sites are suppressed since they would involve high energy costs in the limit $U \gg|t|$. The second term, with $J=4 t^{2} / U$ has a form of an effective exchange interaction of the spins of electrons on neighbouring ions $\left(\mathrm{Cu}^{2+}\right.$ in $\mathrm{CuO}_{2}$ planes, or centres of the wave functions of the Zhang-Rice singlets [5] in a more realistic description).

In the case of half-filling, i.e. one electron per lattice site when the eigenvalues of $n_{i,-\sigma}=1$ and $n_{i, \sigma}=0$ for all lattice sites $i$, the Hamiltonian $\mathcal{H}_{t-J}$ reduces 
to the Heisenberg type Hamiltonian with the exchange ("kinetic exchange" [1]) interaction $J$ favouring antiparallel alignment of spins of electrons on neighbouring sites. For small deviations from the half-filling situation we have a low concentration of carriers (holes) $x=1-\left\langle n_{i,-\sigma}\right\rangle$. The motion of holes is highly correlated with states of the all electrons in system in a quite nontrivial way as we can guess from the above-mentioned intuitive picture. Helpful in understanding complexities of the correlated motions of the holes is the concept of spin-charge separation. First, we have to implement the fundamental restriction on the $t-J$ Hamiltonian (1) by eliminating in calculations states with double occupancies of any site $i$, i.e. eigenvalues of $n_{i \sigma} n_{i,-\sigma}$ must be 0 for each site $i$. There are a few ways of eliminating "unphysical" states with double occupancies, from simple heuristic approaches [2, 3] to a formal derivation of a Hamiltonian equivalent to (1) in the space of physical states, i.e. states with single occupations only [6]. In the present lecture we shall use the simple exact procedure conserving the timereversal symmetry proposed in [7] and applied to the spin polarons problem in [8]. The physical space of only single-occupied space is spanned on the three possible local states for each lattice site $i$ : $|i \sigma\rangle,(\sigma=+$ or -$),|i 0\rangle$ corresponding to an electron at the site $i$ with the spin projection $\sigma$ and a hole at $i$. A physical insight into the intricate correlated motion of electric carriers in the AF planes can be obtained by the idea of spin-charge separation.

For each lattice site there are defined spinless fermionic holon operators $e_{i}, e_{i}^{+}$and (formal)spin $1 / 2$ operators $\boldsymbol{S}_{i}$. There are two possible holon states, no-holon $|i 0\rangle_{h}$ and one holon $|i 1\rangle_{h}$ ones. By definition $e_{i}^{+}|i 0\rangle_{h}=|i 1\rangle_{h}$, $e_{i}|i 1\rangle_{h}=|i 0\rangle_{h}$. Also, there are two spin states at $i$, spin-up $|i \uparrow\rangle_{s}$ and spin-down $|i \downarrow\rangle_{s}$ ones. The local Hilbert space being the tensor product of the holon and spin states thus contains $2 \times 2=4$ states. The physical local space consists of 3 states for each $i$ : $|i \sigma\rangle_{s},|i 0\rangle_{s}$. Therefore, to map the local physical space into the holon-spin space it is necessary to devise a way of eliminating one redundant local state. It was proposed [7] to define local spin states as the superpositions $|i s\rangle_{s}=(1 / \sqrt{2})\left(|i \uparrow\rangle_{s}+\mathrm{e}^{\mathrm{i} \varphi}|i \downarrow\rangle_{s}\right)$ and $|i \bar{S}\rangle_{s}=(1 / \sqrt{2})\left(|i \downarrow\rangle_{s}-\mathrm{e}^{-\mathrm{i} \varphi}|i \uparrow\rangle_{s}\right)$ which are orthogonal to each other, $\varphi$ is an arbitrary phase. The physical space is mapped into the holon-(formal)spin space [7] by the following equivalences: $|i \sigma\rangle \rightarrow|i 0\rangle_{h}|i \sigma\rangle_{s},|i 0\rangle \rightarrow|i 1\rangle_{h}|i s\rangle_{s}$. It can be shown, after implementing the mapping that matrix elements involving the state $|i \bar{S}\rangle_{s}$ all vanish. That is the reason for discarding the state $|i \bar{S}\rangle_{s}$ as unphysical and we finally have one-to-one correspondence of the local physical three states $|i \sigma\rangle,|0 \sigma\rangle$ into the three holon-spin states. The procedure of expressing the $t-J$ Hamiltonian (1) in terms of holon $e_{i}$ and spin operators $\boldsymbol{S}_{i}$ can be easily done if we realize that the operators in the kinetic term in (1) are the Hubbard operators $X_{i \sigma}=c_{i \sigma}\left(1-n_{i,-\sigma}\right)$ and their complex conjugate $X_{i \sigma}^{+}$. They have the simple properties, $X_{i \sigma}|i \sigma\rangle=|i 0\rangle, X_{i \sigma}^{+}|i 0\rangle=|i \sigma\rangle$. The representation $X_{i \sigma}=|i 0\rangle\langle i \sigma|$ can easily be used to express $X_{i \sigma}$ in holon $e_{i}$ 
and (formal)spin operators $\boldsymbol{S}_{i}$ from the correspondence of states [7]:

$X_{i \sigma}=|i 0\rangle\langle i \sigma|\rightarrow| i 1\rangle_{h}|i S\rangle_{s}\left\langle\left. i \sigma\right|_{h}\langle i 0|\right.$.

Since $e_{i}^{+}|i 0\rangle_{h}=|i 1\rangle_{h}, S_{i}^{+}|i \downarrow\rangle_{s}=|i \uparrow\rangle_{s}, S_{i}^{-}|i \uparrow\rangle_{s}=|i \downarrow\rangle_{s}$

$|i 0\rangle\langle i \sigma| \rightarrow X_{i \uparrow}=e_{i}^{+} \frac{1}{\sqrt{2}}\left(S_{i}^{+} S_{i}^{-}+\mathrm{e}^{\mathrm{i} \varphi} S_{i}^{-}\right)$

and, similarly

$$
X_{i \downarrow}=e_{i}^{+} \frac{1}{\sqrt{2}}\left(S_{i}^{+}+\mathrm{e}^{\mathrm{i} \varphi} S_{i}^{-} S_{i}^{+}\right) .
$$

It is easy to verify from the commutation rules of the local electron operators $c_{i \sigma}, c_{i \sigma}^{+}$the following relations:

$$
n_{i \sigma}=X_{i \sigma}^{+} X_{i \sigma}, \quad s_{i}^{+}=X_{i \uparrow}^{+} X_{i \downarrow}
$$

and those derivable from (4). Now from (3) and (4), using the known commutation rules for spin-1/2 operators $S_{i}^{+}, S_{i}^{-}, S_{i}^{z}$ we get e.g.

$$
\begin{aligned}
& n_{i \uparrow}=\left(1-e_{i}^{+} e_{i}\right) S_{i}^{+} S_{i}^{-}, \quad n_{i \downarrow}=\left(1-e_{i}^{+} e_{i}\right) S_{i}^{-} S_{i}^{+}, \\
& n_{i}=n_{i \uparrow}+n_{i \downarrow}=1-e_{i}^{+} e_{i}
\end{aligned}
$$

and

$$
s_{i}^{ \pm}=\left(1-e_{i}^{+} e_{i}\right) S_{i}^{ \pm}, \quad s^{z}=\left(1-e_{i}^{+} e_{i}\right) S_{i}^{z} .
$$

From (5a) it is evident, why we called $\boldsymbol{S}_{i}$ the formal local spin operators as distinct from the "true" local electron spin operators $\boldsymbol{s}_{i}$ : only if there is no holon at the lattice site $i$, i.e. the eigenvalue of $1-e_{i}^{+} e_{i}$ is $1, \boldsymbol{S}_{i}$ has a real physical meaning; in the other case in the presence of the holon at $i, 1-e_{i}^{+} e_{i}$ is 0 and $\boldsymbol{S}_{i}$ is spurious. Finally, the $t-J$ Hamiltonian (1) expressed in the terms of holon $e_{i}, e_{i}^{+}$ and (formal)spin-1/2 operators $\boldsymbol{S}_{i}$ has the form [7]

$$
\begin{aligned}
\mathcal{H}= & -t \sum_{\langle i, j\rangle} e_{i} e_{j}^{+} \frac{1}{2}\left(f_{i j}+g_{i j}\right) \\
& +\frac{1}{2} J \sum_{\langle i j\rangle}\left(1-e_{i}^{+} e_{i}\right)\left(\boldsymbol{S}_{i} \cdot \boldsymbol{S}_{j}-\frac{1}{4}\right)\left(1-e_{j}^{+} e_{j}\right),
\end{aligned}
$$

where

$$
\begin{aligned}
f_{i j} & =2 \boldsymbol{S}_{i} \cdot \boldsymbol{S}_{j}+\frac{1}{2} \\
g_{i j} & =S_{i}^{+}\left(\frac{1}{2}+S_{j}^{z}\right)+\left(\frac{1}{2}-S_{i}^{z}\right) S_{j}^{z}+S_{i}^{-}\left(\frac{1}{2}-S_{j}^{z}\right)+\left(1+S_{i}^{z}\right) S_{j}^{z} .
\end{aligned}
$$

The sums in (6) are over nearest neighbouring sites $\langle i, j\rangle$.

Earlier a slightly simpler expression for the $t-J$ Hamiltonian in spin-charge separate space was derived [6], in which $f_{i j}$ stands simply for $(1 / 2)\left(f_{i j}+g_{i j}\right)$ in (6). However, while (6) acting on any contribution of the unphysical local states $|i \bar{S}\rangle_{s}$ gives exactly zero, due to a compensation of terms coming from $f_{i j}$ and $g_{i j}$, that is not true if only $f_{i j}$ are present so using this simpler form of the Hamiltonian there remains a need to eliminate somehow unphysical states from calculations. 
The choice $\varphi=\pi / 2$ or $-\pi / 2$ for the phase results in the Hamiltonian (6) having the time-reversal symmetry.

\section{Spin polaron}

As already mentioned, in the case of half-filling, $\left\langle e_{i}^{+} e_{i}\right\rangle=0$ for all $i$, the $t-J$ model Hamiltonian (6) is reduced to the standard spin-1/2 Heisenberg exchange Hamiltonian, which for some crystal lattices and for temperatures below a critical value has an AF spin ordering. Since the $\mathrm{CuO}_{2}$ planes form a quadratic lattice belonging to the class of bipartite lattices, the discussion to follow will be restricted to such a case. A bipartite lattice can be divided into two sublattices, say A and B, such that for a site $j$ belonging to the sublattice A all its nearest neighbours are from the sublattice B and vice versa. In order to deal with AF bipartite lattice it is convenient to use a transformed coordinate systems with the local $z$-axis in the points $i \in \mathrm{B}$ rotated by $180^{\circ}$. Such transformation can be done by the operator [9] $T=\prod_{l} T_{l}$ where $T_{l}=1$ for $l=j \in \mathrm{A}$ and $T_{l}=\left(1-e_{i}^{+} e_{i}\right)\left(S_{i}^{+}+S_{i}^{-}\right)+e_{i}^{+} e_{i}$ for $l=i \in \mathrm{B}$. It is easy to check that applying $T$ to $\mathcal{H}$, (6) results in replacing $f_{i j} \rightarrow \tilde{g}_{i j}, g_{i j} \rightarrow \tilde{f}_{i j}$, in which $S_{i}^{ \pm} \rightarrow S_{i}^{\mp}, S_{i}^{z} \rightarrow-S_{i}^{z}$ for $i \in \mathrm{B}$.

In the exchange part $\mathcal{H}_{J}$ of $\mathcal{H} \equiv \mathcal{H}_{t}+\mathcal{H}_{J},(6)$, rewritten as

$$
\begin{aligned}
\mathcal{H}_{J} & =\frac{1}{2} J \sum_{\langle i, j\rangle}\left(\boldsymbol{S}_{i} \cdot \boldsymbol{S}_{j}-\frac{1}{4}\right)-J \sum_{\langle i, j\rangle} e_{i}^{+} e_{i} \boldsymbol{S}_{i} \cdot \boldsymbol{S}_{j}\left(1-e_{j}^{+} e_{j}\right) \\
& +\frac{1}{4} J \sum_{\langle i, j\rangle} e_{i}^{+} e_{i}\left(1-e_{j}^{+} e_{j}\right)-\frac{1}{2} J \sum_{\langle i, j\rangle} e_{i}^{+} e_{i}\left(\boldsymbol{S}_{i} \cdot \boldsymbol{S}_{j}-\frac{1}{4}\right) e_{j}^{+} e_{j},
\end{aligned}
$$

the first term is the Heisenberg exchange Hamiltonian as for undoped system of real spins antiferromagnetically coupled, let us consider it as an unperturbed Hamiltonian $\mathcal{H}_{0}$. For low concentration of holes $x=\left\langle e_{i}^{+} e_{i}\right\rangle \ll 1$, which we assume, the last contribution to $\mathcal{H}_{J}(7)$ can be neglected because the possibility of finding two holons on neighbouring lattice sites $i, j$ is small. By the same arguments the third term in (7), denoted as $\mathcal{H}_{h}$, can be approximated by the expression

$$
\mathcal{H}_{h}=\frac{1}{4} \bar{J} \sum_{\langle i, j\rangle} e_{i}^{+} e_{j},
$$

where $\bar{J}=J\left\langle\left(1-e_{i}^{+} e_{j}\right)\right\rangle=J(1-x)$. The second term in $(7)$, denoted as $\mathcal{H}_{h-S}$ below, can be simplified by the following arguments.

In the second term of the right hand side (r.h.s.) of Eq. (7) a fictitious spin $e_{i}^{+} e_{i} \boldsymbol{S}_{i}$ of a hole is interacting with real spins $\left(1-e_{j}^{+} e_{j}\right) \boldsymbol{S}_{j}$ of neighbouring electrons. The local Hilbert space of the variable $\boldsymbol{S}_{i}$ at the site $i$ occupied by the hole is constructed from two states $|i s\rangle_{s}$ and $|i \bar{s}\rangle_{s}$, from which only the former one gives non-zero matrix elements of physical quantities. In particular, as indicated above $\mathcal{H},(7)$, acting on a wave function containing $|i \bar{s}\rangle_{s}$, i.e. belonging to the unphysical spin space, will give zero. Therefore, it was proposed [8] to average the unphysical spin in $e_{i}^{+} e_{i} \boldsymbol{S}_{i}$ over the physical state $|i s\rangle_{s}$, i.e. to replace $e_{i}^{+} e_{i} \boldsymbol{S}_{i}$ by 
$e_{i}^{+} e_{i s}\left\langle i s\left|\boldsymbol{S}_{i}\right| i s\right\rangle_{s}$. As a result the second term of the r.h.s. (7) is replaced by the following expression:

$$
\mathcal{H}_{h-s}=-\frac{1}{4} J \sum_{\langle i, j\rangle} e_{i}^{+} e_{i}\left(\mathrm{e}^{\mathrm{i} \varphi} S_{j}^{-}+\mathrm{e}^{-\mathrm{i} \varphi} S_{j}^{+}\right)\left(1-e_{j}^{+} e_{j}\right)
$$

For a low concentration of holes $x \ll 1, J\left(1-e_{j}^{+} e_{j}\right)$ can be approximated by $\bar{J}$ as argued above.

The first term in (7), the exchange energy $\mathcal{H}_{\mathrm{ex}}^{0}$, is the unperturbed Hamiltonian. For a bipartite lattice it has an AF ground state and by applying the transformation $T$ it takes the form

$$
\tilde{\mathcal{H}}_{\mathrm{ex}}^{0}=T \mathcal{H}_{\mathrm{ex}}^{0} T^{-1}=\frac{J}{4} \sum_{\langle i, j\rangle}\left(S_{i}^{+} S_{j}^{+}+S_{i}^{-} S_{j}^{-}-S_{i}^{z} S_{j}^{z}-1 / 2\right) .
$$

The standard procedure for diagonalizing in the linear spin wave (LSW) approximation $\tilde{\mathcal{H}}_{\mathrm{ex}}^{0}$ is to express $S_{i}^{ \pm}, S_{i}^{z}$ in terms of (AF-) magnon boson operators (for details see e.g. [10]): $S_{l}^{-} \cong a_{l}, S_{l}^{+} \cong a_{l}^{+}, S_{l}^{z}=a_{l}^{+} a_{l}-1 / 2$ and expanding $a_{l}$ into the Fourier components, $a_{l}=N^{-1 / 2} \sum_{q} \mathrm{e}^{\mathrm{i} \boldsymbol{q} \cdot \boldsymbol{R}_{l}} \alpha_{\mathrm{q}}, \ldots$, where $\alpha_{q}, \alpha_{q}^{+}$are a set of bosons (the unfolded Brillouin zone (UBZ) is used with $N$ standing for the number of lattice sites and also the number of points in UBZ). $\tilde{\mathcal{H}}_{\text {ex }}^{0}$ expressed in $\left(\alpha_{q}, \alpha_{q}^{+}\right)$contains products $\alpha_{q}^{+} \alpha_{-q}^{+}+\alpha_{q} \alpha_{-q}$ and $\alpha_{q}^{+} \alpha_{-q}$, so to diagonalize $\tilde{\mathcal{H}}_{\mathrm{ex}}^{0}$ the Bogolubov type transformation to a new set of bosons $\left(\zeta_{q}, \zeta_{q}^{+}\right)$is employed, $\alpha_{q}=u_{q} \zeta_{q}+v_{q} \zeta_{-q}^{+}$, where $u_{q}=\cosh \vartheta_{q}, v_{q}=\sinh \vartheta_{q}$, and tanh $2 \vartheta_{q}=-\gamma_{q}, \gamma_{q}=(1 / 2)\left(\cos q_{x}+\cos q_{y}\right)$. Finally, $\tilde{\mathcal{H}}_{\mathrm{ex}}^{0}=\sum_{q} \omega_{q}\left(\zeta_{-}^{+} q \zeta_{q}+1 / 2\right)$ is expressed in terms of normal modes - $\mathrm{AF}$ magnons, having energies $\omega_{q}=2 J \sqrt{1-\gamma_{q}^{2}}$. The same transformations are applied to $\mathcal{H}_{s-h},(8)$, so for $\tilde{\mathcal{H}}_{J}=T \mathcal{H}_{J} T^{-1}$ we get

$$
\tilde{\mathcal{H}}_{J}=\sum_{q} \omega_{q}\left(\zeta_{q}^{+} \zeta_{q}+\frac{1}{2}\right)+\bar{J} \sum_{i} e_{i}^{+} e_{i}+\sum_{q}\left(F_{q} \zeta_{q}+F_{q}^{+} \zeta_{q}^{+}\right),
$$

where $F_{q}=-N^{-1 / 2} \bar{J} \gamma_{q}\left(u_{q}+v_{q}\right) \rho_{q}$ and $\rho_{q}=\sum_{l} e_{l}^{+} e_{l} \mathrm{e}^{\mathrm{i} \boldsymbol{q} \cdot \boldsymbol{R}_{l}}$. The third term in (10) describes the energy of interactions of magnons $\left(\zeta_{q}, \zeta_{q}^{+}\right)$with the fluctuations of the holons density $\rho_{q}$. Such interactions are well known in the theory of lattice polarons. The standard method, like in the polaron theory, is to apply the so-called shift transformation [11] $\zeta_{q} \rightarrow U \zeta_{q}=\zeta_{q}+f_{q}$ to the Hamiltonian $\tilde{\mathcal{H}}_{J}$ in order to eliminate the linear terms in (10). The unitary transformation has the form [12]

$$
U=\exp \left(\sum_{q}\left(F_{q}^{+} \zeta_{q}^{+}-F_{q} \zeta_{q}\right) / \omega_{q}\right)
$$

(it is easy to check that $f_{q}$ has to be taken as $\left.-F_{q} / \omega_{q}\right)$. The transformed Hamiltonian $\tilde{\mathcal{H}}_{J} \rightarrow U \tilde{\mathcal{H}}_{J} U^{-1}$ is 


$$
\tilde{\mathcal{H}}_{J}=\sum_{q} \omega_{q}\left(\zeta_{q}^{+} \zeta_{q}+\frac{1}{2}\right)+J_{1} \sum_{i} e_{i}^{+} e_{i},
$$

where

$$
J_{1}=\bar{J}\left[1-N^{-1} \sum_{q} \frac{\bar{J} \gamma_{q}^{2}}{\omega_{q}}\left(u_{q}^{2}+v_{q}^{2}\right)\right] .
$$

Now we have to express the transport term $\mathcal{H}_{t}$ in $\mathcal{H},(6)$, in the same magnon formalism. In the physical space the forms of $\mathcal{H}_{t}$ with $(1 / 2)\left(f_{i j}+g_{i j}\right)$ or with $f_{i j}$ [6] are equivalent, so to simplify the formulae we shall use $f_{i j}$ in what follows and for the arbitrary phase $\varphi$ we shall take $\varphi=0$. To the $T \mathcal{H}_{t} T^{-1}$ we apply the LSW and then the shift transformation $U$ to get

$$
\tilde{\mathcal{H}}_{t}=-t \sum_{\langle i, j\rangle} U e_{i} e_{j}^{+}\left(a_{i}^{+}+a_{j}\right) U^{-1} .
$$

The factor $U\left(a_{i}^{+}+a_{j}\right) U^{-1}$ can be easily expressed as a linear combination of magnon operators $\zeta_{q}, \zeta_{q}^{+}$, whereas [8]

$$
U e_{i} e_{j}^{+} U^{-1}=e_{i} e_{j}^{+} \Phi_{i j},
$$

where

$$
\begin{aligned}
& \Phi_{i j}=\exp \left(N^{-1 / 2} \sum_{q} A_{i j}^{q}\left(\zeta_{q}-\zeta_{-q}^{+}\right)\right), \\
& A_{i j}^{q}=-\bar{J}\left(\mathrm{e}^{\boldsymbol{q} \cdot \boldsymbol{R}_{i}}-\mathrm{e}^{\mathrm{i} \boldsymbol{q} \cdot \boldsymbol{R}_{j}}\right) \gamma_{q}\left(u_{q}+v_{q}\right) / \omega_{q} .
\end{aligned}
$$

The factor $\Phi_{i j}$ in (15), reminiscent of the so-called band narrowing factor in the lattice polarons theory, leads in general to a complicated pattern of multimagnon processes. To propose an intuitive picture of the motion of holons in AF planes we recall that average of $f_{i j}$, or $f_{i j}+g_{i j}$ over the Néel AF state is exactly zero. This observation corresponds to the fact already mentioned that in the Néel state holon is localized. If we take into account fluctuations of spins, i.e. magnons, we get a nonzero average $N^{-1} \sum_{q}\left(\sqrt{1-\gamma_{q}^{2}}-1\right)$ of $f_{i j}\left(\right.$ or $\left.f_{i j}+g_{i j}\right)$ over a true ground state involving zero point motion of spins. Thus holon can move, since it is assisted by magnons. The average of $\Phi_{i j}$ over the true AF ground state is

$$
\begin{aligned}
\left\langle\Phi_{i j}\right\rangle & =\exp \left(-N^{-1} \sum_{q}\left(\bar{J} \gamma_{q} / \omega_{q}\right)^{2}\left(1-\gamma_{q}^{2}\right)^{-1 / 2}\right. \\
& \left.\times\left[1+\gamma_{q} \cos \boldsymbol{q} \cdot\left(\boldsymbol{R}_{i}-\boldsymbol{R}_{j}\right)\right]\right)
\end{aligned}
$$

(the average over the Néel state $\left\langle\Phi_{i j}\right\rangle \equiv 1$ ). The factor $\left\langle\Phi_{i j}\right\rangle$ can be interpreted as narrowing the holon band width $\sim t$ to $t\left\langle\Phi_{i j}\right\rangle$. In trying to estimate the band narrowing factor there appear difficulties due to divergent sums over wave vectors $\boldsymbol{q}$ in our two-dimensional system. The reason is that only at half-filling, i.e. when there are no holons, $x=0$, the long-range AF order exists in 2D. For finite $x$ there exists only a short range AF order with spin-spin correlation length of the order of $a / \sqrt{x}[13]$, where $a$ is the lattice constant. Thus summations over wave vectors in the system with a short-range order have to exclude the region at the centres 
of the Brillouin zone for $q<q_{\mathrm{c}} \simeq \pi \sqrt{x} / a$. For the nearest neighbours $(i, j),\left\langle\Phi_{i j}\right\rangle$ changes from 0.22 for $x=0.01$, to 0.74 for $x=0.05$, but extrapolates to 0 for $x \rightarrow 0$ (cf. [8]). For doping at the level of a few percent, as in the case of $\mathrm{CuO}_{2}$ planes in real systems of interest, the narrowing of the holon band is thus not very dramatic. However, there are other fundamental questions connected with $\Phi_{i j}$. If $\Phi_{i j}$ is expanded into magnon operators it leads to multimagnon processes increasingly difficult to deal with quantitatively.

The factor $\Phi_{i j}$ can be expanded into a series $\Phi_{i j}=1+\sum(\ldots)\left(\zeta_{q}-\zeta_{q}^{+}\right)+\ldots$ For $\Phi_{i j} \cong 1$ the transport Hamiltonian $\tilde{\mathcal{H}}_{t}$ is reduced to $\tilde{\mathcal{H}}_{t} \cong-t \sum_{\langle i, j\rangle} e_{i} e_{j}^{+} U \times$ $\left(a_{i}^{+}+a_{j}\right) U^{+}$corresponding to one-magnon type interactions with holons. The one-magnon processes are expected to be dominant and they will be studied in detail. The effect of higher order processes can be estimated by approximating the expression $\left(\Phi_{i j}-1\right) U\left(a_{i}^{+}+a_{j}\right) U^{+}$in $\tilde{\mathcal{H}}_{t},(14),(15)$, by its average over the quantum AF ground state $|0\rangle_{\mathrm{AF}}$. The approximate correction $w=$ $\mathrm{AF}\left\langle 0\left|\left(\Phi_{i j}-1\right) U\left(a_{i}^{+}+a_{j}\right) U^{+}\right| 0\right\rangle_{\mathrm{AF}}$ was estimated in [8] for nearest neighbours $\langle i, j\rangle$, $w$ depends only on the holon concentration and is roughly $w \cong x / 2$ for $x \ll 1$.

The holon operators $e_{i}$ are expanded into their Fourier components $h_{k}$ (obviously, satisfying, like $e_{i}, e_{i}^{+}$, fermion commutation rules)

$$
e_{i}=N^{-1 / 2} \sum_{k} \mathrm{e}^{\mathrm{i} \boldsymbol{k} \cdot \boldsymbol{R}} h_{k} .
$$

The transport Hamiltonian takes now the form

$$
\tilde{\mathcal{H}}_{t}=N^{-1 / 2} \sum_{k q} P(\boldsymbol{k}, \boldsymbol{q})\left(h_{k}^{+} h_{k-q} \zeta_{q}+\text { h.c. }\right)+\sum_{k}\left(-t w \gamma_{k}\right) h_{k}^{+} h_{k},
$$

where $P(\boldsymbol{k}, \boldsymbol{q})=4 t\left(\gamma_{k-q} u_{q}+\gamma_{k} v_{q}\right)$, c.f. [3]. From $\tilde{\mathcal{H}}_{t},(18)$, it is evident that holon movements are accompanied by disturbances in the AF order. The dominant one-magnon term in $\tilde{\mathcal{H}}_{t}$ corresponding to scatterings of holon with creation or annihilation of an AF magnon was extensively studied in literature (cf. e.g. [3]). The second term in $\tilde{\mathcal{H}}_{t}$, (18), having the form of an effective holon kinetic energy approximately accounts for multimagnon processes accompanying motions of holes.

Now the total Hamiltonian $\tilde{\mathcal{H}}=\tilde{\mathcal{H}}_{t}+\tilde{\mathcal{H}}_{J}$ takes the form

$$
\begin{aligned}
\tilde{\mathcal{H}}= & \sum_{k} E_{k} h_{k}^{+} h_{k}+\sum_{q} \omega_{q}\left(\zeta_{q}^{+} \zeta_{q}+1 / 2\right) \\
& +N^{-1 / 2} \sum_{k q} P(\boldsymbol{k}, \boldsymbol{q})\left(h_{q}^{+} h_{k-q} \zeta_{q}+\text { h.c. }\right),
\end{aligned}
$$

where

$$
E_{k}=-t w \gamma_{k}+J_{1}
$$

$\tilde{\mathcal{H}}$ in (19) has the well-known form of a Hamiltonian in the standard polaron problem, the difference is that the kinetic energy $E_{k}$ of carriers is now a small correction only. Since $J / t$ is quite small (a typical value for cuprates is about $J / t \cong 0.3)$ also the magnon energy $\omega_{q} \sim J$ is lower than the interaction energy 
$\sim P(\boldsymbol{k}, \boldsymbol{q}) \sim t$. Thus, unlike the case of classical lattice polaron we have a problem of a strong-coupling and a simple perturbational treatment is not sufficient now. While dealing with spin polarons strong-coupling problem it was proposed to use the self-consistent Born approximation (SCBA) [2, 3]. The validity of the SCBA was checked by numerical simulations (see e.g. [4]) and is considered as a firm basis of the spin polaron theory.

For calculating the spin polaron spectrum the single-particle holon Green function is considered

$$
G(\boldsymbol{k}, \omega)=\left\langle 0\left|h_{k}(\omega-\tilde{\mathcal{H}})^{-1} h_{k}^{+}\right| 0\right\rangle,
$$

where $|0\rangle$ denotes the ground state of the holon-magnon system, i.e. the product of the holon vacuum state and the quantum AF ground state of the magnon subsystem. The Green function calculated in SCBA $G(\boldsymbol{k}, \omega)$ has the form

$$
G(\boldsymbol{k}, \omega)=1 /\left[\omega-E_{k}-\sum(\boldsymbol{k}, \omega)\right],
$$

where the self-energy is given by [3]

$$
\begin{aligned}
& \sum(\boldsymbol{k}, \omega)=N^{-1} \sum_{q}|P(\boldsymbol{k}, \boldsymbol{q})|^{2} \\
& \quad \times\left[\omega-\omega_{q}-E_{k-q}-\sum\left(\boldsymbol{k}-\boldsymbol{q}, \omega-\omega_{q}\right)\right]^{-1} .
\end{aligned}
$$

The calculation of $G(\boldsymbol{k}, \omega),(22),(23)$, in SCBA corresponds to summing up to all orders contributions from the interactions $\sim|P|^{2}$ which correspond to graphs with no crossings of interaction lines [3]. The self-energy is calculated numerically from

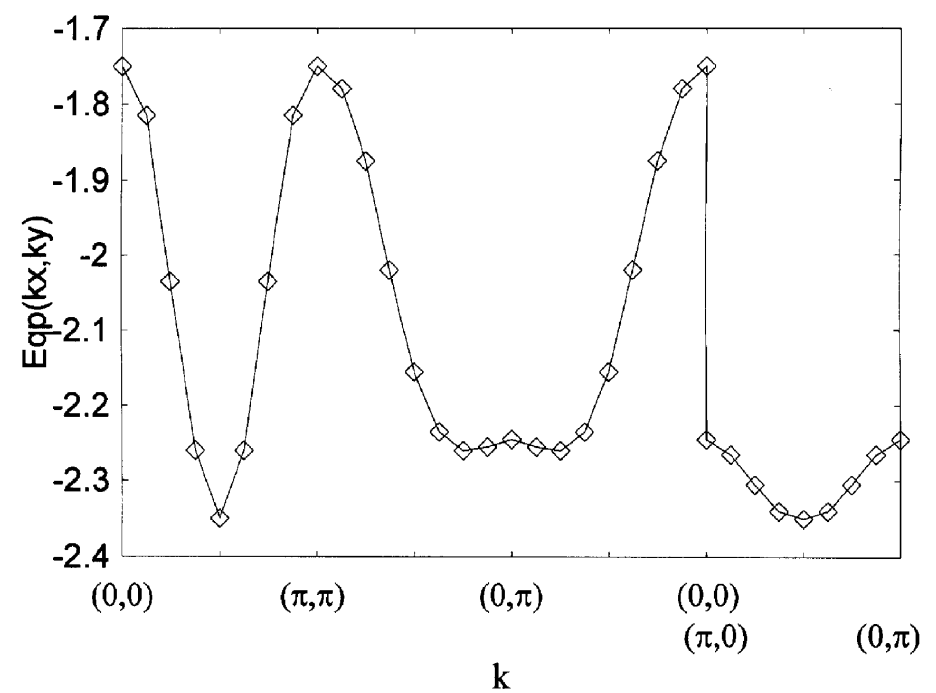

Fig. 1. Spin polaron energy $E_{\mathrm{qp}}$ dispersion vs. $\boldsymbol{k}$ for the directions $\Gamma X((0,0)-(\pi, \pi))$, $X M((\pi, \pi)-(\pi, 0)), \quad M \Gamma((\pi, 0)-(0,0)), \quad$ and $M M^{*}((\pi, 0)-(0, \pi)), \quad$ computed for $J / t=0.3$ for the quadratic lattice. The energy is measured in units of $t$, the wave vector $\boldsymbol{k}$ - in the inverse lattice constant. 
the self-consistency equation (23) for a mesh of $\boldsymbol{k}$-points covering the Brillouin zone of the quadratic lattice. The quality of interest is the spectral function $F(\boldsymbol{k}, \omega)=(1 / \pi) \operatorname{Im} G(\boldsymbol{k}, \omega+\mathrm{i} \varepsilon)$. The spectral function $F(\boldsymbol{k}, \omega)$ of holons strongly interacting with magnons consists of a low-energy peak of a spectral weight varying with the $\boldsymbol{k}$ and a broad basically incoherent spectrum. The lowest energy peak is attributed to a quasiparticle - a bound state of a hole dressed in a cloud of magnons, i.e. to a spin polaron. Thus the positions $\Omega$ of the lowest energy peaks, on the energy axis $\omega$ for given wave vector $\boldsymbol{Q}$ determine the polaron energy $\Omega(\boldsymbol{Q})$. An example of the energy dispersion curve for the spin polarons, calculated for $J=0.3 t$ (the value estimated as realistic for YBCO [4]) and $x=0.05$ is given in Fig. 1.

The spin polaron concept was introduced in efforts at understanding properties of the high $T_{\mathrm{c}}$ superconductors $\left(\mathrm{HT}_{\mathrm{c}} \mathrm{S}\right)$. The polaron dispersion relation, a simplest example presented in Fig. 1, and also obtained by extensive numerical simulations [4], was confronted with measurements of the dispersion by angleresolved photoemission [14]. The model with only nearest neighbours hoppings of holes is not confirmed by experiments. The spectrum changes significantly if also intra lattice hoppings $t^{\prime}$ are taken into account [8]. It was shown that a model with hoppings up to the third neighbours can be brought into account with experimental data if the hopping parameters $t, t^{\prime}$, and $t^{\prime \prime}$ as well as $J$ are chosen accordingly [15].

\section{Interaction of spin polarons}

The concept of spin polarons is helpful in understanding complexities of motion of charge carriers within $\mathrm{CuO}_{2}$ planes in cuprates. The obvious question is whether there exists a mechanism of pairing of spin polarons and whether they are relevant for forming a superconductivity state in the cuprates. The problem of the existence of bound states of two holes in AF planes was studied both by numerical simulations [16-18] and by the several analytical approaches with approximations not easy to verify [19-21]. Here we shall try to calculate the energy of a pair of interacting holons, using an equivalent formalism for the energy of a single holon and to compare this energy with a sum of energies of corresponding two independent holons. For the two-holon states we cannot neglect the last term in $\mathcal{H}_{J},(7)$, which while vanishing for a single hole state, negligible for low concentration of holes, now can play a role as a contact interaction of holes. The last term in $\mathcal{H}_{J}$ will be simplified by approximating $\boldsymbol{S}_{i} \cdot \boldsymbol{S}_{j}-1 / 4$ by its average value over the quantum AF ground state. Therefore, now the total Hamiltonian including the contact term $V_{h h}$ will read $\mathcal{H}=\tilde{\mathcal{H}}+V_{h h}$, where

$$
V_{h h}=N^{-1} \sum_{k k^{\prime} q} W \gamma_{q} h_{k-q}^{+} h_{k^{\prime}+q}^{+} h_{k^{\prime}} h_{k}
$$

where $W=2 J_{\mathrm{AF}}\left\langle 0\left|\left(\boldsymbol{S}_{i} \cdot \boldsymbol{S}_{j}-1 / 4\right)\right| 0\right\rangle_{\mathrm{AF}}$. For quadratic lattice $W \cong-1.16 J$ (cf. e.g. Eq. (28) in [8]). To find the energy of a holon pair, say $\left(\boldsymbol{k}, \boldsymbol{k}^{\prime}\right)$, the 
two-particle Green function is like (21),

$$
G\left(\boldsymbol{k}, \boldsymbol{k}^{\prime}, \omega\right)=\left\langle 0\left|h_{k^{\prime}} h_{k}(\omega-\mathcal{H})^{-1} h_{k}^{+} h_{k^{\prime}}\right| 0\right\rangle,
$$

here $\mathcal{H}=\tilde{\mathcal{H}}+V_{h h}$ as discussed above. Again, $G\left(\boldsymbol{k}, \boldsymbol{k}^{\prime}, \omega\right)$ is calculated in the SCBA

$$
G\left(\boldsymbol{k}, \boldsymbol{k}^{\prime}, \omega\right)=\left[\omega-E_{k}-E_{k^{\prime}}-\sum\left(\boldsymbol{k}, \boldsymbol{k}^{\prime}, \omega\right)\right]^{-1},
$$

where the two-particle self-energy $\sum\left(\boldsymbol{k}, \boldsymbol{k}^{\prime}, \omega\right)$ is determined self-consistently by the equation [22]

$$
\begin{aligned}
& \sum\left(\boldsymbol{k}, \boldsymbol{k}^{\prime}, \omega\right)=N^{-1} \sum_{q}\left[P_{k q}^{2} G\left(\boldsymbol{k}-\boldsymbol{q}, \boldsymbol{k}^{\prime}, \omega-\omega_{q}\right)+P_{k^{\prime} q}^{2} G\left(\boldsymbol{k}, \boldsymbol{k}^{\prime}-\boldsymbol{q}, \omega-\omega_{q}\right)\right. \\
& \left.+2 W^{2} N^{-1}\left(\gamma_{k-q}-\gamma_{k^{\prime}-q}\right)^{2} G\left(\boldsymbol{q}, \boldsymbol{q}-\boldsymbol{k}-\boldsymbol{k}^{\prime}, \omega-\omega_{q}\right)\right] .
\end{aligned}
$$

Equations (27) and (26) for the self-energy of the pair of interesting holons can be solved numerically by iterations similarly as for the single holon case. The calculations were done for the parameter value $J / t=0.3$, relevant for real systems [22]. An example of the results is presented in Fig. 2. The energy of a pair of inter-

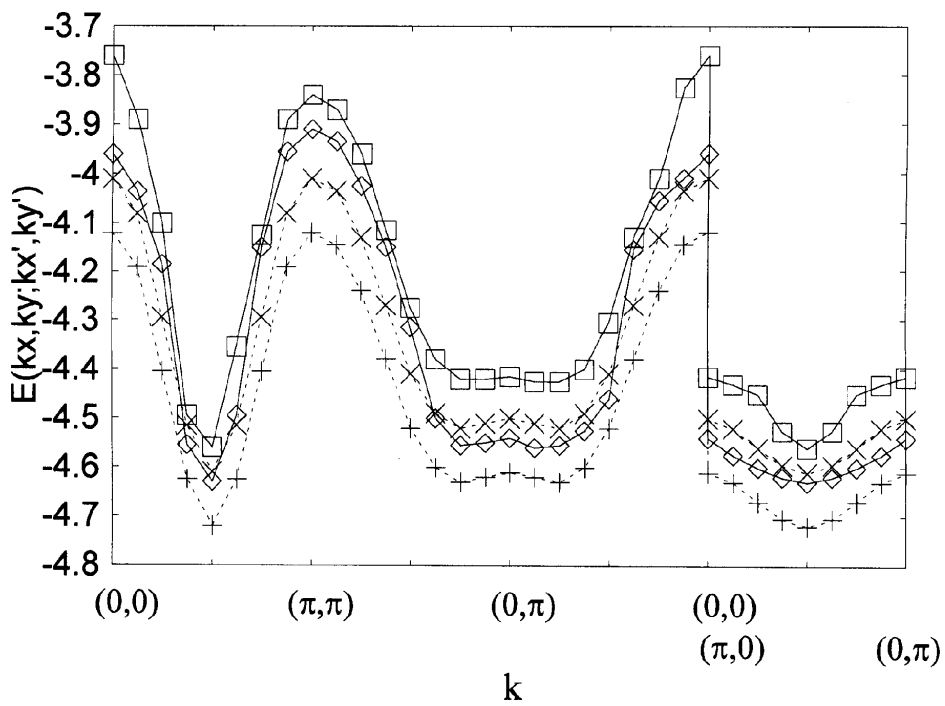

Fig. 2. Energy dispersion curves for a free pair of spin polarons $\boldsymbol{k}$ and $\boldsymbol{k}^{\prime}, E_{1}(\boldsymbol{k})+E_{1}\left(\boldsymbol{k}^{\prime}\right)$ (dotted lines) and for a pair of interacting spin polarons, $E_{2}\left(\boldsymbol{k}, \boldsymbol{k}^{\prime}\right)$ (full lines) calculated for $\boldsymbol{k}^{\prime}=(0, \pi)$ (squares and crosses), and for $\boldsymbol{k}^{\prime}=(\pi / 2, \pi / 2)$ (diamonds and "+"), for $\boldsymbol{k}$ directions, as in Fig. 1, computed for $J / t=0.3$. Energies are measured in units of $t$, points are calculated, lines are guides to the eye.

acting spin polarons $\boldsymbol{k}, \boldsymbol{k}^{\prime}, E_{2}\left(\boldsymbol{k}, \boldsymbol{k}^{\prime}\right)$ is compared with the sum of the energies of "free" polarons $E_{1}(\boldsymbol{k})+E_{1}\left(\boldsymbol{k}^{\prime}\right)$, for $\boldsymbol{k}$ along symmetry lines in the Brillouin zone and for the two characteristic values of $\boldsymbol{k}^{\prime}$, i.e. $\boldsymbol{k}^{\prime}=(0,0)$ and $(\pi / 2, \pi / 2)$. The energy difference $\Delta=E_{2}\left(\boldsymbol{k}, \boldsymbol{k}^{\prime}\right)-E_{1}(\boldsymbol{k})-E_{2}\left(\boldsymbol{k}^{\prime}\right)$ is positive for all $\boldsymbol{k}$ and chosen 
values $\boldsymbol{k}^{\prime}$, as it is evident from Fig. 2. In fact, $\Delta>0$ for all pairs of values $\left(\boldsymbol{k}, \boldsymbol{k}^{\prime}\right)$, not only for those exemplified in Fig. 2. Extensive numerical calculations have shown [22] that for $J / t$ smaller than a critical value of the order of 0.5 for any pair of values $\left(\boldsymbol{k}, \boldsymbol{k}^{\prime}\right)$ the energy difference $\Delta$ is positive, therefore a hypothetical bound state of two spin polarons would not be stable. Only for values $J / t>0.6$, out of a realistic range, there are some regions in the space $\left(\boldsymbol{k}, \boldsymbol{k}^{\prime}\right)$, where $\Delta$ could be negative. It can be thus concluded that pairing of spin polarons seems to be a remote possibility for realistic situations.

\section{References}

[1] K.A. Chao, J. Spałek, A.M. Oleś, J. Phys. C 10, L277 (1977).

[2] S. Schmitt-Rink, C.M. Varma, A.E. Ruckeisen, Phys. Rev. Lett. 10, 2793 (1988).

[3] G. Martinez, P. Horsch, Phys. Rev. B 44, 317 (1991).

[4] E. Dagotto, Rev. Mod. Phys. 66, 763 (1994).

[5] F.C. Zhang, T.M. Rice, Phys. Rev. B 37, 3759 (1988).

[6] Y.R. Wang, M.J. Rice, Phys. Rev. B 49, 4360 (1994).

[7] J. Loos, Phys. Rev. B 53, 12556 (1996).

[8] J. Loos, J. Morkowski, Physica C 298, 17 (1998).

[9] J.L. Richard, V.Yu. Yushankai, Phys. Rev. B 47, 1103 (1999).

[10] F. Keffer, in: Encyclopedia of Physics, Ed. S. Flügge, Springer, Berlin 1966, p. 1.

[11] I.G. Lang, Yu.A. Firsov, Sov. Phys.-JETP 16, 1301 (1963).

[12] J. Morkowski, Acta Phys. Pol. A 97, 101 (2000).

[13] N. Furukawa, M. Imada, J. Phys. Soc. Jpn. 61, 3331 (1992).

[14] B.O. Wells, Z.-X. Shen, A. Matsuura, D.M. King, M.A. Kastner, M. Greven, R.J. Birgeneau, Phys. Rev. Lett. 74, 964 (1995).

[15] O.P. Sushkov, G.A. Sawatzky, R. Eder, H. Eskes, Phys. Rev. B 56, 11769 (1997).

[16] Y. Hasegawa, D. Poilblanc, Phys. Rev. B 40, 9035 (1989).

[17] T. Barnes, A.E. Jacobs, M.P. Kovarik, W.G. Nacready, Phys. Rev. B 45, 256 (1991).

[18] M. Bonisegni, E. Manousakis, Phys. Rev. B 47, 11897 (1993).

[19] M.I. Kuchiev, O.P. Sushkov, Physica $C$ 218, 197 (1993).

[20] V.I. Belinicher, A.L. Chernyshev, V.A. Shubin, Phys. Rev. B 56, 3381 (1997).

[21] A.V. Dotsenko, Phys. Rev. B 67, 6917 (1998).

[22] J. Morkowski, J. Magn. Magn. Mater. 226-230, 444 (2001). 